\title{
THE RELATIONSHIP BETWEEN CLINICAL CLASSIFICATION OF CATARACT AND LENS HARDNESS
}

\author{
P. HEYWORTH ${ }^{1}$, G. M. THOMPSON ${ }^{1}$, H. TABANDEH ${ }^{1}$ and S. McGUIGAN ${ }^{2}$ \\ London
}

\begin{abstract}
SUMMARY
'Hardness' of the cataractous lens is one of the major factors influencing the suitability of a patient for phacoemulsification. Surgeons should be able to estimate hardness when choosing patients for this method of cataract extraction. The aims of the study were to investigate the relationship between the clinical appearance of cataracts and their hardness. Ninety-one lenses were studied from patients undergoing endocapsular cataract extraction. Lenses were assessed using a classification based on the Lens Opacities Classification System II and hardness was assessed using an automated lens guillotine. The degree of nuclear sclerosis and the age of the patient were found to influence lens hardness. Regression analysis showed that $56 \%$ of the variation in hardness could be explained in terms of age and the degree of nuclear sclerosis. The presence of posterior subcapsular and cortical cataract did not influence hardness. These findings confirm our clinical suspicions and enable surgeons to make a more objective analysis of hardness prior to phacoemulsification.
\end{abstract}

Phacoemulsification is becoming an increasingly popular method of cataract surgery. However, there is an increased complication rate during the learning curve and this is a major factor dissuading many surgeons from undertaking phacoemulsification on a routine basis.

Factors influencing the 'difficulty' of the operation include lens hardness (as suggested by brunescence), deep-set eyes, shallow anterior chamber and poorly dilated pupils. Lens hardness is probably the most important single factor and is associated with increased phaco time and power. This makes for a difficult operation overall and is associated with increased morbidity. By avoiding hard lenses surgeons can expect a smoother and safer operation. An association between clinical appear-

From: 'Department of Ophthalmology, St. George's Hospital, London, UK; ${ }^{2}$ Department of Mental Health Sciences, St. George's Hospital Medical School, London, UK.

Correspondence to: Graham M. Thompson, FRCS, FRCOphth, Department of Ophthalmology, St. George's Hospital, London SW17 0QT, UK. ance of the cataract and its hardness has long been suspected, cataract surgeons recognising that nuclear brunescence equates with hardness. To our knowledge there are no objective studies in the literature confirming this.

We investigated the relationship between the clinical classification of cataract and hardness.

\section{SUBJECTS AND METHODS}

Following instillation of G. phenylephrine $10 \%$ and G. cyclopentolate $1 \%, 91$ patients with cataract underwent slit lamp examination in order to classify the type and degree of cataract prior to surgery. Patients with mature cataract were excluded from the study. A classification system based on the Lens Opacities Classification System II $^{1.2}$ was used. This system was chosen for its simplicity and reproducibility. It does not require photography and is suitable for clinical situations. Three main types of cataract were recognised, namely nuclear, posterior subcapsular and cortical. Each type was then compared with a standard set of colour photographs. The nuclear element of the cataract was evaluated by colour (range $0-4$ ) and by opalescence (range 0-4). These two factors were assessed with the slip lamp beam at $45^{\circ}$ to the visual axis. Colours ranged from the faintest hint of green $(=1)$ to dark brown $(=4)$. Opalescence was measured according to the extent to which the cataract prevented the transmission of light. Posterior subcapsular cataracts were assessed using retroillumination by the red reflex and a grading ( $0-3)$ was made according to the area of posterior pole involved. Cortical cataracts were evaluated $(0-4)$ using retro-illumination according to the area involved.

Lenses extracted using the endocapsular technique were placed in a moist chamber for a maximum of 2 hours before being assessed for hardness on an automated guillotine (Fig. 1). This machine incorporates a cutting system activated by a switch which starts a digital timer and allows water to flow from a large reservoir into a receiver. The receiver is attached to the end of a swinging arm balanced on a fulcrum. Beneath the fulcrum a sharp

Eуe (1993) 7, 726-730 
razor blade exerts increasing cutting force on the lens, which is supported upon a bevelled block. When the lens has been bisected a trip switch is activated stopping the digital timer and the further flow of water. The flow rate of water from the large reservoir remains constant throughout the investigation. Knowing the volume of water required to bisect the lens allows the total force in newtons to be calculated.

\section{RESULTS}

The study was confined to a comparison of clinical appearance of the cataract and patient's age with lens hardness. Ninety-one lenses were studied. Seventy-two cataracts were thought to be senile in origin, 17 were associated with diabetes and 7 had other cataract risk factors such as chronic use of systemic steroids, retinitis pigmentosa and congenital lens opacities. In the young patient group (age less than 60 years), 6 cataracts were associated with risk factors such as diabetes mellitus, strong family history or chronic ocular disease and in 7 subjects there were not any identifiable risk factors. The mean age was 73.5 years (range $30-95$ years). The average force required to bisect the lenses was $1.10 \mathrm{~N}$.

\section{Age (Fig. 2, Table I)}

The mean force for the under-60s group was $0.80 \mathrm{~N}$ and for the over-80s group was $1.38 \mathrm{~N}$. There is a tendency for

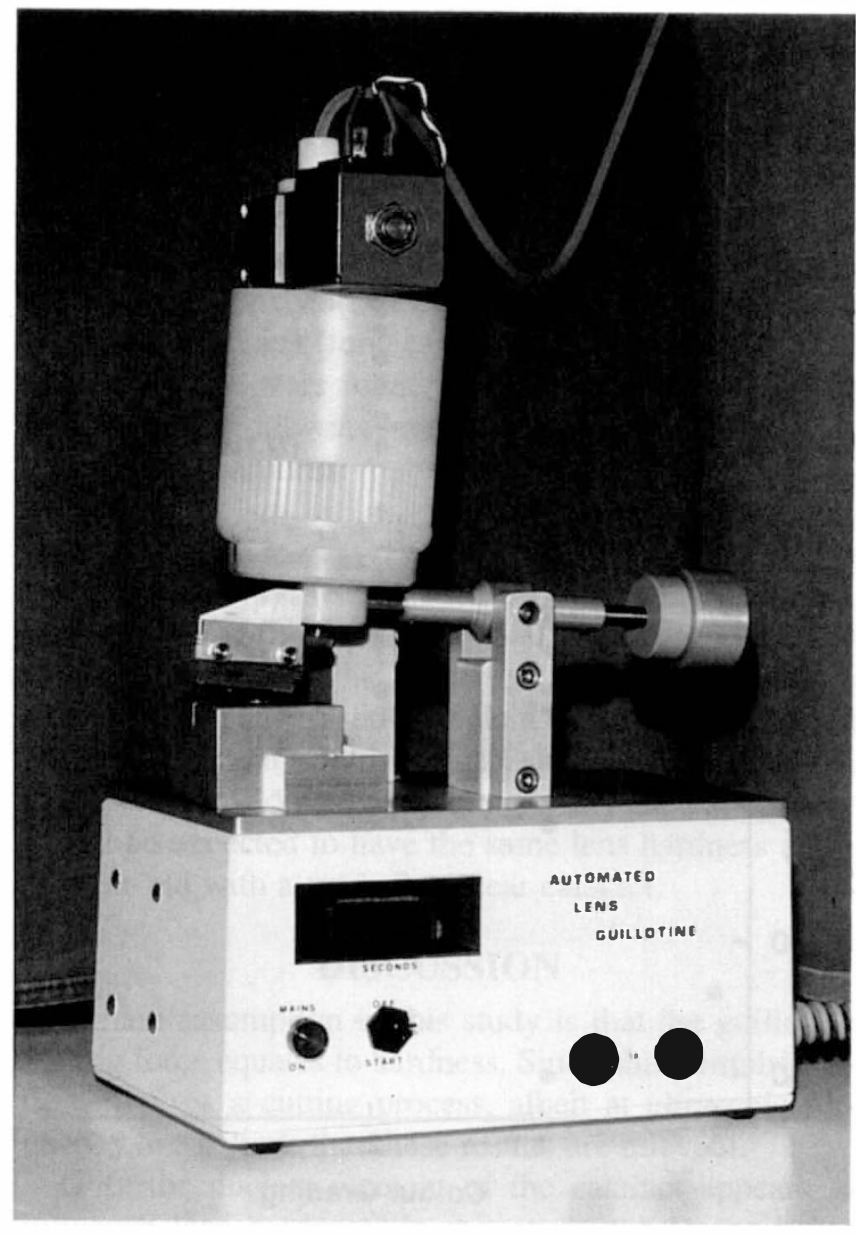

Fig. 1. An automated lens guillotine. force and therefore hardness to increase with advancing age, although there remains a wide range within each group. The range of hardness between the softest and hardest lenses is a factor of 30. The linear tendency for increasing hardness on a logarithmic scale suggests accelerating hardness with advancing age.

\section{Nuclear Colour and Opalescence}

In this series the grading of colour and opalescence were related, the difference between colour and opalescence being within one grade in $97 \%$ of cases. Only colour grading will be considered (Fig. 3, Table II). There is a fourfold difference in mean force between the grade 1 colour group $($ mean $=0.58 \mathrm{~N})$ and grade 4 colour group $($ mean $=2.16 \mathrm{~N})$. When considering the effects of both age and nuclear colour (Fig. 4) the softest lenses tend to have the lower colour gradings and the harder lenses have the higher gradings. Colour and age appear to be independent factors since the mean age of each colour group remains within 6 years of the overall mean (Fig. 5). Colour is therefore not purely a reflection of age.

\section{Cortical and Subcapsular Lens Opacities (Tables III, IV)}

Posterior subcapsular and cortical types of cataract do not

Table I. Lens hardness according to age group

\begin{tabular}{lcccc}
\hline Age $(\mathrm{yr})$ & $\begin{array}{c}\text { Mean force } \\
(\mathrm{N})\end{array}$ & Frequency & $\begin{array}{c}\text { Max. force } \\
(\mathrm{N})\end{array}$ & $\begin{array}{c}\text { Min. force } \\
(\mathrm{N})\end{array}$ \\
\hline$<60$ & 0.80 & 13 & 2.87 & 0.10 \\
$61-70$ & 0.87 & 20 & 3.12 & 0.24 \\
$71-80$ & 1.12 & 31 & 2.51 & 0.24 \\
$>80$ & 1.38 & 27 & 2.54 & 0.23 \\
\hline
\end{tabular}

Table II. Lens hardness according to the nuclear colouration

\begin{tabular}{lccc}
\hline Colour & Mean force $(\mathrm{N})$ & Mean age $(\mathrm{yr})$ & Frequency \\
\hline 1 & 0.58 & 69 & 41 \\
2 & 1.26 & 79 & 27 \\
3 & 1.67 & 77 & 15 \\
4 & 2.16 & 76 & 8 \\
\hline
\end{tabular}

Table III. Mean force necessary to bisect the lens according to the extent of cortical involvement

\begin{tabular}{lccc}
\hline Cortical LO & Mean force (N) & Mean age (yr) & Frequency \\
\hline 0 & 1.34 & 72 & 49 \\
1 & 0.94 & 74 & 18 \\
2 & 0.87 & 78 & 10 \\
3 & 0.50 & 80 & 8 \\
4 & 0.82 & 62 & 5 \\
\hline LO, lens opacity. & &
\end{tabular}

Table IV. Mean force necessary to bisect the lens according to the extent of posterior subcapsular lens opacification (PSCLO)

\begin{tabular}{lccc}
\hline PSCLO & Mean force $(\mathrm{N})$ & Mean age $(\mathrm{yr})$ & Frequency \\
\hline 0 & 1.27 & 76 & 44 \\
1 & 0.56 & 75 & 10 \\
2 & 1.12 & 74 & 15 \\
3 & 0.92 & 67 & 20 \\
\hline
\end{tabular}




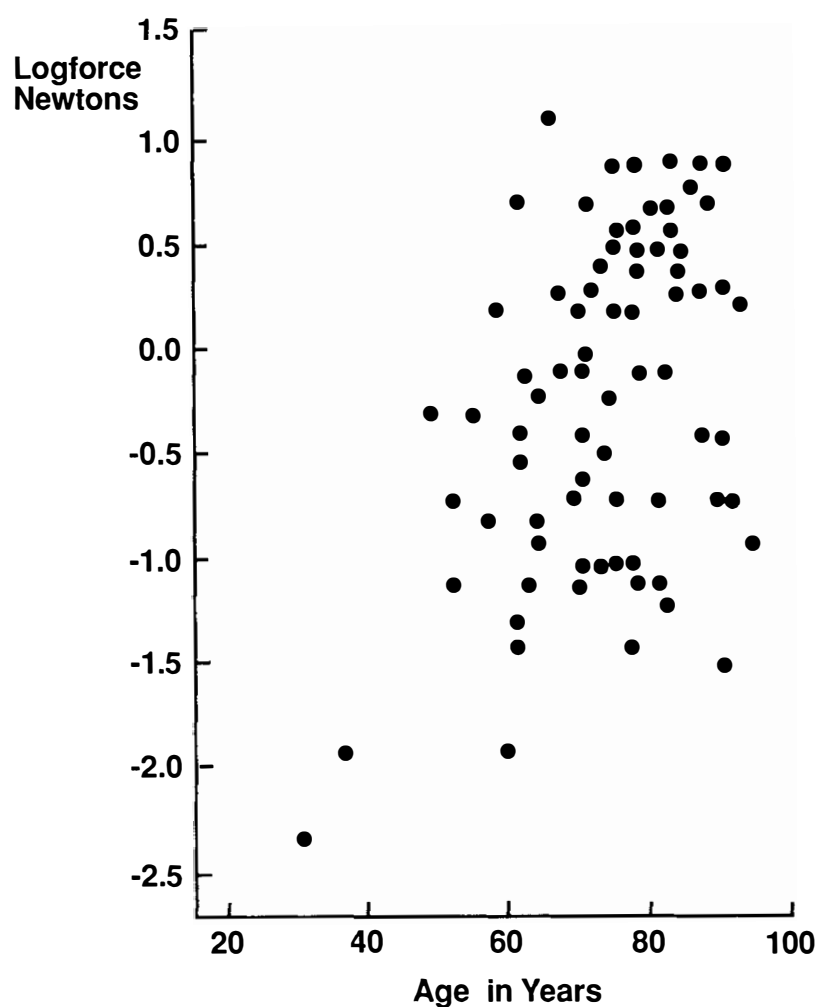

Fig. 2. Log force versus age.

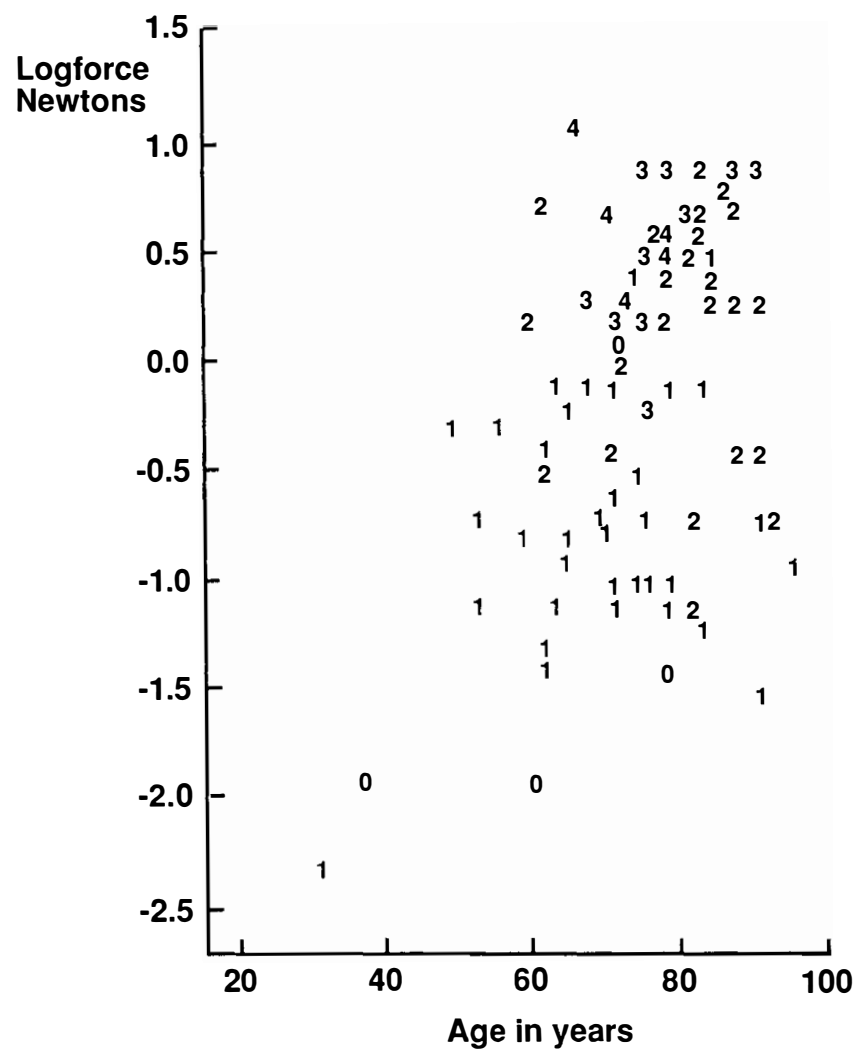

Fig. 4. Log force versus age and nuclear colour.

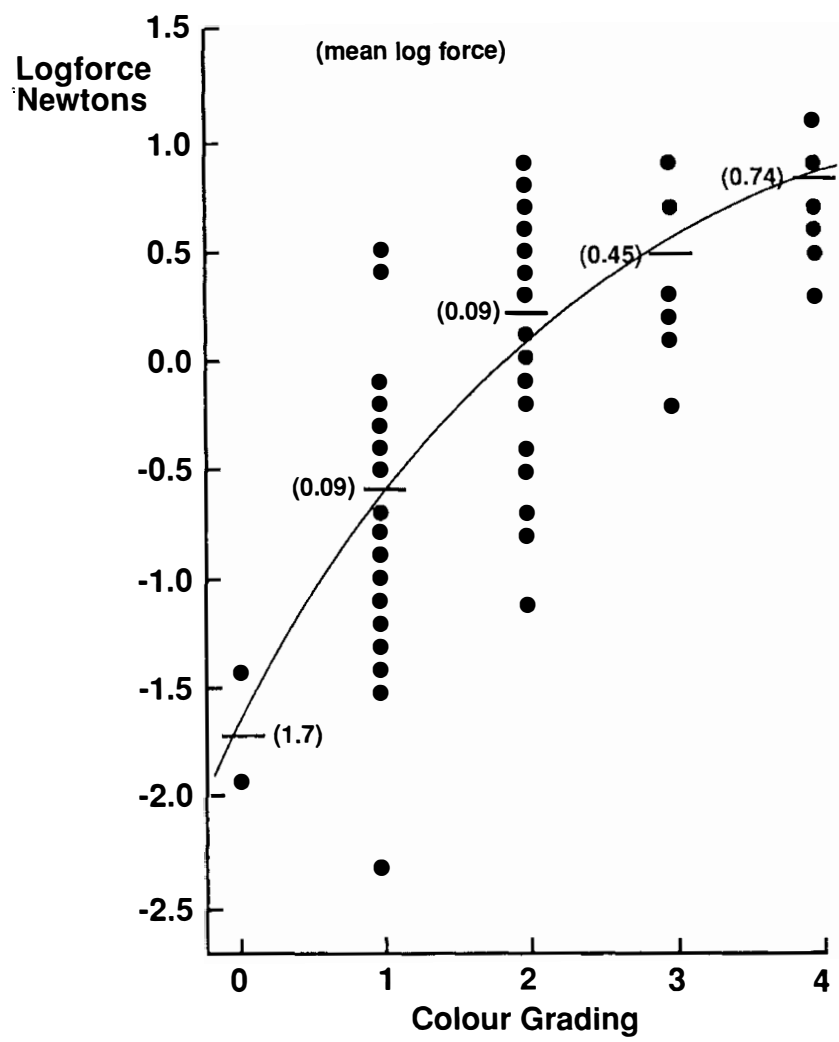

Fig. 3. Log force versus colour.

\section{Age in Years}

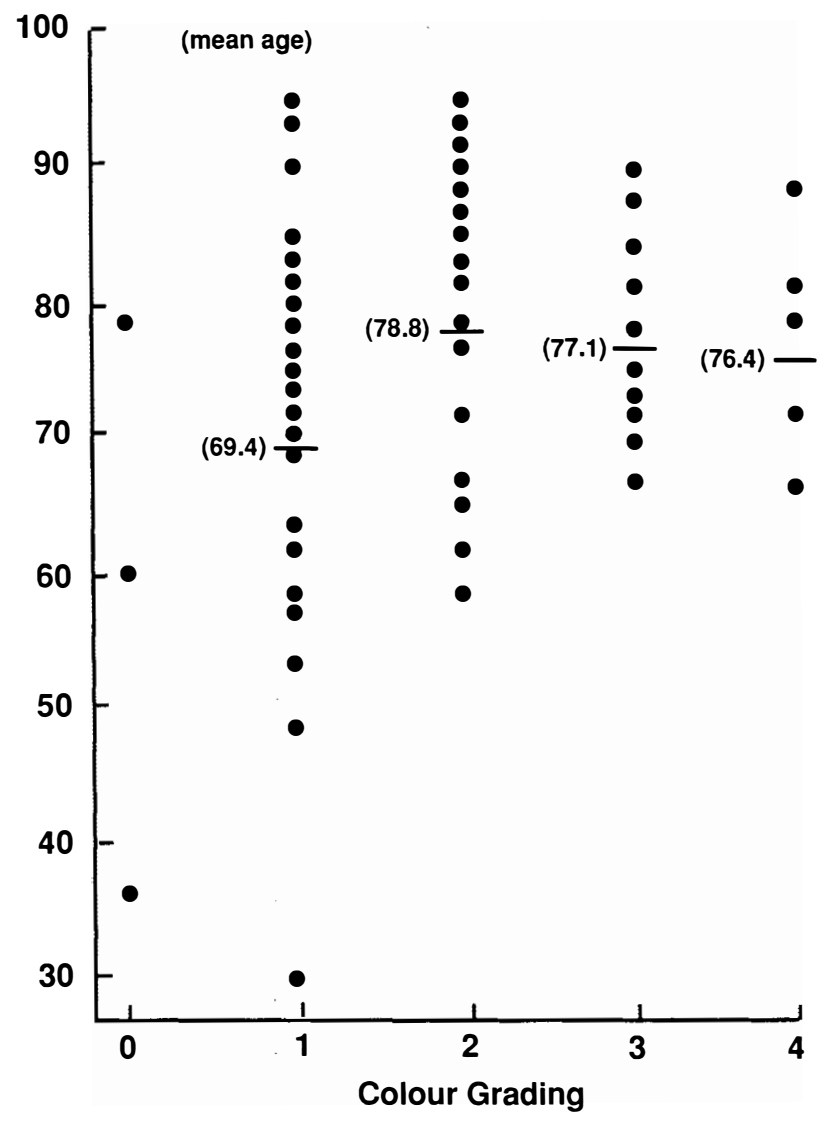

Fig. 5. Age versus colour. 


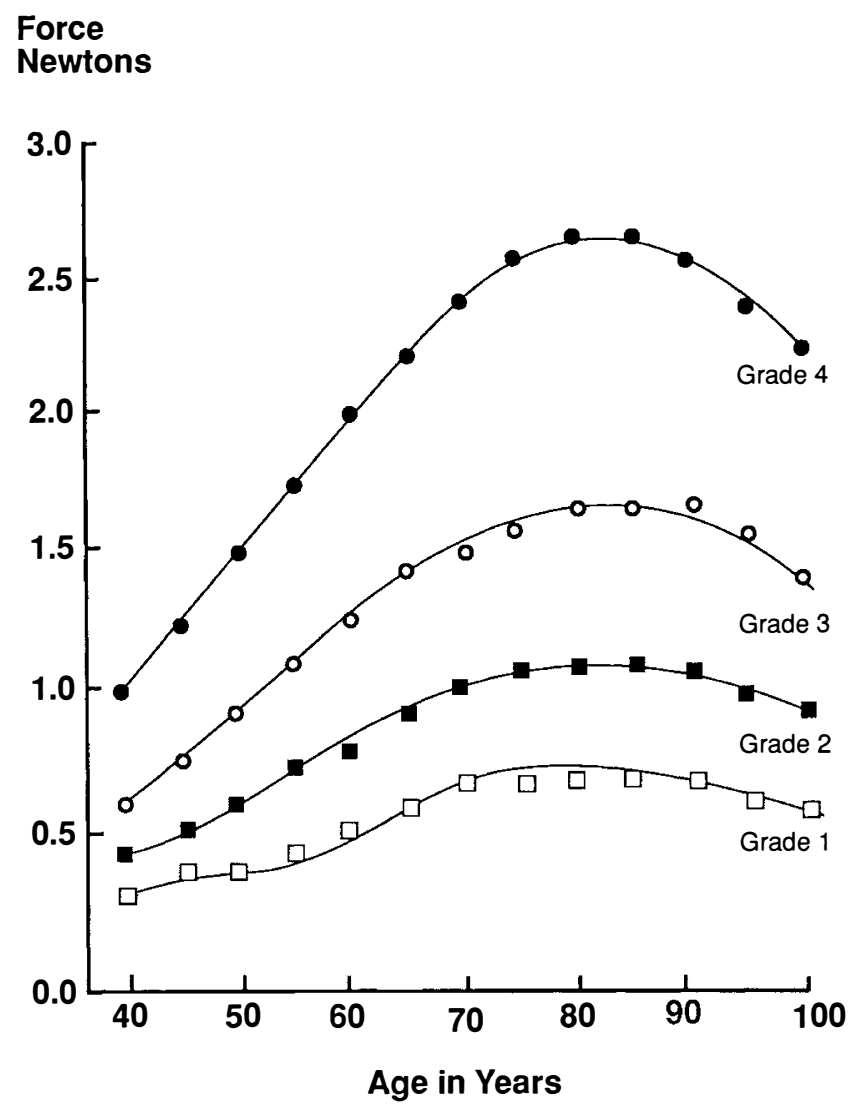

Fig. 6. Force versus age for various nuclear colours.

appear to influence lens hardness. The mean force necessary to bisect the lens does not follow the same pattern as that of nuclear sclerosis, with gradings being randomly scattered. The mean force for a lens with a clear cortex was $1.34 \mathrm{~N}$ and for grade 4 cortical involvement it was $0.82 \mathrm{~N}$.

Regression analysis was applied to the data obtained. Using lens hardness (force) as the dependent variable, lens colour and age were found to be important explanatory variables. The following model explained $56 \%$ of the variation in lens hardness:

$$
\log _{\mathrm{e}}(\text { force })=\alpha+\beta \text { Age }+\gamma(\text { Age })^{2}+\delta \text { Colour }
$$

where $\alpha=-4.49$ (SE 1.13), $\beta=+0.087$ (SE 0.03), $\gamma=-0.0005$ (SE 0.0002), $\delta=+0.462$ (SE 0.06) and adjusted $R^{2}=0.56$.

The model can be used to explain hardness for different degrees of nuclear sclerosis and age (Fig. 6). It can be seen that a 40 -year-old patient with a grade 4 nuclear cataract would be expected to have the same lens hardness as an 80 -year-old with a grade 2 nuclear cataract.

\section{DISCUSSION}

The main assumption in this study is that the guillotine cutting force equates to hardness. Since phacoemulsification involves a cutting process, albeit at ultrasonic frequency, we believe that these results are relevant.

Only the nuclear element of the cataract appears to influence lens hardness. In this study the lenses being assessed were extracted using the endocapsular technique in which the majority of the cortical and posterior subcapsular elements are left behind as soft lens matter for aspiration. Clinically it is accepted that this material is the softest element of the lens although we did not objectively measure it on the guillotine. It is therefore not surprising that we did not find the cortical and posterior subcapsular elements to influence lens hardness. It could, however, be argued that these elements have a direct or indirect influence upon adjacent nuclear hardness. We found no such effect and can conclude that the cortical and posterior subcapsular elements are not useful markers for hardness.

Little has been written about the precise biochemical and physical changes that influence lens hardness. Factors such as protein changes, the compactness of lens fibres and water content are probably relevant.

Age

With advancing age the nucleus increases in size and the lens fibres become compacted and compressed. Animal studies also demonstrate an increase in the intermolecular disulphide cross-linking during ageing. These bonds compact the $\gamma$-crystallin molecules thereby promoting closer packing and hence hardening. ${ }^{3}$ In the human lens there is a cortex-to-core increase in insoluble lens protein which becomes more apparent around the onset of presbyopia. ${ }^{4}$ Our results show a tendency for hardness to increase with age, although there is still a large range within each group. This finding may be a result of increasing lens fibre compactness and increasing proportions of insoluble lens protein occurring in the ageing lens.

\section{Colour}

The precise nature of the yellow colouration of the lens is unknown; however, it correlates well with many biochemical changes. Colour appears to be mostly associated with an increase in water-insoluble lens proteins and in particular the urea-insoluble subfraction. ${ }^{5.6}$ The change to a pigmented insoluble protein occurs as a result of photooxidation of tyrosine and tryptophan. ${ }^{6}$ With continued alteration of lens proteins the colour becomes more intense. These changes are not seen in the cortical region of a nuclear cataract nor in the nuclear region of a normal lens. This study demonstrates a relationship between lens hardness and the degree of nuclear sclerosis as assessed by colouration and opalescence. The underlying biochemical and structural changes that are responsible for the development of colouration would also appear to confer hardness upon the lens.

In summary, the study confirms a relationship between lens hardness and the clinical appearance of the cataractous lens. We have identified two major markers for lens hardness: (1) the degree of nuclear sclerosis as measured by colour and opalescence and (2) the age of the patient. By considering these factors pre-operatively at the slit lamp the surgeon can choose cases suitable for phacoemulsification.

The authors wish to thank Dr. C. Slingsby of Birkbeck College, London, for her useful comments, Dr. John Terry of Gillette UK, and the Department of Medical Physics, St. George's Hospital, London. 
Key words: Cataract classification, Lens hardness, Nuclear sclerosis, Phacoemulsification.

\section{REFERENCES}

1. Chylack LT, Leske MC, McCarthy D, Khu P, Kashiwagi T, Sperduto R. Lens opacities classification system II (LOCS II). Arch Ophthalmol 1989;107:991-7.

2. Chylack LT, Lee MR, Tung WH, Cheng HM. Classification of human senile cataractous change by the American Co-operative Cataract Research Group (CCRG) method. I. Instru- mentation and technique. Invest Ophthalmol Vis Sci 1983;24:424-31.

3. Hum TP, Augusteyn RC. The nature of disulphide bonds in rat lens proteins. Curr Eye Res 1987;6:1103-8.

4. Li LK, Roy D, Spector A. Changes in lens protein in concentric fractions from individual normal human lenses. Curr Eye Res 1986;5:127-35.

5. Pirie A. Colour and solubility of the proteins of human cataracts. Invest Ophthalmol 1968;7:634-50.

6. Zigman S. Eye lens colour: formation and function. Science 1971;171:807-9. 